\title{
Collembola Poduromorpha do litoral de Maricá, Rio de Janeiro, Brasil
}

\author{
Liliane Henriques Fernandes ${ }^{1,2} \&$ Maria Cleide de Mendonça ${ }^{1}$
}

${ }^{1}$ Departamento de Entomologia, Museu Nacional. Quinta da Boa Vista, São Cristóvão, 20940-040 Rio de Janeiro, Brasil. E-mail: liliane@acd.ufrj.br, mcleide@acd.ufrj.br

2 Bolsista do CNPq.

\begin{abstract}
Collembola Poduromorpha from the littoral of Maricá, Rio de Janeiro, Brazil. New species and new occurrences of Collembola Poduromorpha in the littoral of Maricá, state of Rio de Janeiro are recorded. From the 20 species collected, four are described and illustrated: Mesaphorura maricaensis sp. nov. (Onychiuridae); Brachystomella ceciliae sp. nov. (Brachystomellidae); Arlesia intermedia sp. nov. and Micranurida fluminensis sp. nov. (Neanuridae). The genus Doutnacia Rusek, 1974 and Micranurida Börner, 1901 and the species Xenylla maritima Tullberg, 1869, Acherontiella globulata Thibaud \& Massoud, 1979, Onychiurus cf. mariapetrae Thibaud, 1993, Friesea reducta Denis, 1931, Pseudachorutes difficilis Denis, 1931 are recorded for the first time to Brazil. The high number of species and the record of the 16 new occurrences in this area shows the scarcity of the taxonomic studies and the elevated biodiversity of the Poduromorpha at sand dunes.
\end{abstract}

KEY WORDS. Brachystomellidae, Hypogastruridae, Neanuridae, Onychiuridae, Tullbergiidae.

RESUMO. São registradas novas espécies e novas ocorrências de Collembola Poduromorpha no litoral de Maricá, estado do Rio de Janeiro. Das 20 espécies coletadas, quatro são descritas e ilustradas: Mesaphorura maricaensis sp. nov. (Onychiuridae); Brachystomella ceciliae sp. nov. (Brachystomellidae); Arlesia intermedia sp. nov. e Micranurida fluminensis sp. nov. (Neanuridae). Os gêneros Doutnacia Rusek, 1974 e Micranurida Börner, 1901 e as espécies Xenylla maritima Tullberg, 1869, Acherontiella globulata Thibaud \& Massoud, 1979, Onychiurus cf. mariapetrae Thibaud, 1993, Friesea reducta Denis, 1931, Pseudachorutes difficilis Denis, 1931 são registrados pela primeira vez para o Brasil. O elevado número de espécies e o registro de 16 novas ocorrências nesta área mostram a escassez de estudos taxonômicos e a elevada biodiversidade de Poduromorpha na restinga.

PALAVRAS CHAVE. Brachystomellidae, Hypogastruridae, Neanuridae, Onychiuridae, Tullbergiidae.

A Ordem Poduromorpha sensu Borner (1913), é uma das maiores da Classe Collembola e inclui atualmente cerca de 2600 espécies distribuídas em todas as regiões zoogeográficas (Bellinger et al. 1996). As restingas, devido à sua composição topográfica e condições ambientais, propiciam a formação de muitos habitats; flora e fauna ricas e variadas podem ser encontradas nesta região (Silva \& Oliveira 1989). Entretanto, estes ambientes vêm sendo constantemente atingidos pela especulação imobiliária, uma das principais responsáveis pela devastação, que transforma grande parte de suas áreas em fragmentos da vegetação original.

No Brasil, estudos envolvendo os Poduromorpha ainda são escassos, principalmente os relacionados com a fauna das restingas. Atualmente 13 espécies de Poduromorpha são registradas para o litoral brasileiro: Anurida maritima (Guérin, 1838), Halachorutes schusteri Arlé, 1966, Brachystomella agrosa Wray, 1953, Maricaella duna Mendonça \& Fernandes, 1997, Ceratophysella bengtssoni (Agren, 1904), Mesogastrura cf. ojcoviensis (Stach, 1918), Austrogastrura travassosi (Arlé, 1939), Willemia brevispina Hüther, 1962, Friesea cubensis Potapov \& Banasko, 1985, Friesea josei Palacios-Vargas, 1986, Fissuraphorura cubanica Rusek, 1991; Mesaphorura yosii (Rusek, 1967) e Aethiopella littoralis Fernandes \& Mendonça, 2002 (ArLÉ 1966a, MendonçA \& Arlé 1992, Mendonça \& Fernandes 1997, Thibaud \& PalaciosVargas 1999, Fernandes \& Mendonça 2002).

O objetivo deste estudo foi descrever novos táxons e registrar novas ocorrências de Collembola Poduromorpha na Restinga de Itaipuaçu, município de Maricá, Rio de Janeiro.

\section{MATERIAL E MÉTODOS}

A Restinga de Itaipuaçu, $4^{\circ}$ Distrito de Maricá (entre o Canal da Costa e a Praia de Itaipuaçu), situa-se no litoral fluminense a leste da cidade do Rio de Janeiro (22 $57^{\prime} 50^{\prime \prime} \mathrm{S}$ e $\left.42^{\circ} 50^{\prime} 40^{\prime \prime} \mathrm{W}\right)$, sendo parte integrante da grande planície litorânea arenosa quaternária, que ocorre ao longo de toda a costa brasileira (Suguio \& Tessler 1984). A restinga apresenta clima do tipo tropical quente superúmido com subseca (NIMER 1977), com temperaturas médias de $16^{\circ} \mathrm{C}$ a $28^{\circ} \mathrm{C}$ e precipitação anual 
oscilando entre 1000 e $1250 \mathrm{~mm}$, com mínima mensal de $30 \mathrm{~mm}$ e período de seca de abril a agosto (Dorvillé 1995).

Foram realizadas excursões mensais à Restinga de Itaipuaçu entre Maio de 1995 e Abril de 1996 para coleta de solo/folhiço no período compreendido entre 7:00 e 12:00 h. Foram coletadas 180 amostras padronizadas por meio de uma sonda cilíndrica metálica com $4 \mathrm{~cm}$ de profundidade por 10 $\mathrm{cm}$ de diâmetro. As amostras acondicionadas em sacos plásticos etiquetados foram colocadas no laboratório em funis de Berlese-Tullgren para extração da fauna a uma temperatura de $25^{\circ} \mathrm{C}$, durante seis dias. Os espécimens extraídos foram fixados em álcool fervente, e a seguir, triados e identificados sob microscópio estereoscópico e microscópio óptico. O estudo taxonômico de Poduromorpha foi realizado com o auxílio de chaves e literatura especializada (SALMON 1964, MASSOUD 1967, Christiansen \& Bellinger 1980, Palácios-Vargas \& Mejia 1988, Thibaud 1990, Arbea \& Jordana 1991, Christiansen \& Bellinger 1992, LuCiañez \& Simón 1993, Weiner 1996). Todo o material estudado, inclusive holótipos e parátipos, foi depositado na Coleção de Collembola do Museu Nacional, Rio de Janeiro (CM/ MNRJ). No item referente à lista de material examinado empregou-se a seqüência: número do lote, número de exemplares (ex. e exs) e data de coleta.

\section{RESULTADOS}

Foram coletados 14.134 colêmbolos, sendo 5.507 pertencentes a Poduromorpha, distribuídos em cinco famílias, três subfamílias, 15 gêneros e 20 espécies, caracterizadas a seguir.

\section{Hypogastruridae Xenylla maritima Tullberg, 1869}

Material examinado. BRASIL, Rio de Janeiro: Maricá, Restinga de Itaipuaçu, em substratos de dunas e de áreas alagáveis, L.H. Fernandes leg. 526, 83 exs, 532, 3 exs, 533, 21 exs, 534, 12 exs, 535, 149 exs, 536, 27 exs, 539, 14 exs, 27/V/ 95; 545, 25 exs, 547, 8 exs, 548, 4 exs, 550, 3 exs, 551, 92 exs, 552, 12 exs, 553, 1 exs, 554, 1 exs, 23/VII/95; 556, 10 exs, 562, 7 exs, 564, 2 exs, 565, 106 exs, 566, 270 exs, 567, 4 exs, 20/VII/ 95; 576, 1 exs, 579, 1 exs, 585, 3 exs, 586, 12 exs, 587, 56 exs, 588, 2 exs, 31/VIII/95; 598, 44 exs, 600, 3 exs, 601, 8 exs, 603, 95 exs, 27/IX/95; 606, 180 exs, 613, 21 exs, 615, 5 exs, 616, 24 exs, 617, 2 exs, 618, 28 exs, 27/X/95; 628, 12 exs, 629, 3 exs, 630, 7 exs, 631, 772 exs, 632, 158 exs, 633, 2 exs, 634, 24 exs, 24/XI/95; 641, 4 exs, 643, 76 exs, 644, 2 exs, 646, 18 exs, 647, 95 exs, 648, 2 exs, 649, 12 exs, 650, 1 ex., 16/XII/95; 656, 6 exs, 658, 55 exs, 659, 10 exs, 661, 13 exs, 664, 8 exs, 22/I/96; 673, 6 exs, 676, 23 exs, 29/II/96; 686, 156 exs, 688, 53 exs, 689, 8 exs, 690, 12 exs, 691, 5 exs, 25/III/96; 703, 172 exs, 705, 34 exs, 706, 38 exs, 707, 2 exs, 709, 10 exs, 19/IV/96.

Comentários. Nos exemplares de Xenylla maritima coletados em Itaipuaçu verificou-se a presença da cerda $\mathbf{p}_{1}$ na região cefálica dorsal, diferentemente do observado por GAMA (1964).

\section{Acherontiellla globulata Thibaud \& Massoud, 1979}

Material examinado: BRASIL, Rio de Janeiro: Maricá, Restinga de Itaipuaçu, em substratos de dunas, L.H. Fernandes leg. 526, 4 exs, 27/V/95.

Comentários. Este é o primeiro registro do gênero para o Brasil. Os exemplares de Acherontiella globulata mostraram-se concordantes com a descrição de Thibaud \& MAssoud (1979) baseada em exemplares coletados em guano de uma gruta na Ilha de Maria Galante (América Central).

\section{Onychiuridae Onychiurus cf. mariapetrae Thibaud, 1993}

Material examinado: BRASIL, Rio de Janeiro: Maricá, Restinga de Itaipuaçu, em substratos de áreas alagáveis, L.H. Fernandes leg. 568, 1 ex., 20/VII/95; 618, 1 ex., 27/X/95; 632, 1 ex., 634, 10 exs, 24/XI/95; 647, 2 exs, 16/XII/95; 709, 1 ex., 711, 6 exs, 19/IV/96.

Comentários. A única diferença observada nos exemplares de Itaipuaçu diz respeito à fórmula pseudocelar da face ventral dos segmentos abdominais I e III. Segundo Thibaud (1993), os exemplares da série original descritos de Guadalupe, apresentam em cada um dos referidos segmentos, 1+1 pseudocelo, ao passo que os espécimens provenientes da restinga de Itaipuaçu apresentam $2+2$ pseudocelos.

\section{Tullbergiidae}

\section{Mesaphorura amazonica Oliveira \& Thibaud, 1992}

Material examinado. Brasil, Rio de Janeiro: Maricá, Restinga de Itaipuaçu, em substratos de dunas, L.H. Fernandes leg. 638, 5 exs, 639, 1 ex., 16/XII/95; 655, 9 exs, 22/I/96; 683, 11 exs, 684, 6 exs, 685, 1 ex., 25/ III/96; 699, 12 exs, 701, 4 exs, 702, 3 exs, 19/IV/96.

Comentários. Os exemplares de Mesaphorura amazonica concordam em grande parte com a descrição original. No entanto, pequenas diferenças foram observadas no que se refere à quetotaxia dos segmentos abdominais II e III e no tocante a relação unha: espinho anal. No material descrito do Amazonas a cerda $\mathrm{p}_{5}$ apresenta-se em forma de macroqueta e a relação unha:espinho anal é de $2: 1$, ao passo que no material coletado na restinga, esta cerda apresentou-se em forma de microqueta e a relação unha:espinho anal foi de 1:1.

\section{Mesaphorura yosii (Rusek, 1967)}

Material examinado. Brasil, Rio de Janeiro: Maricá, Restinga de Itaipuaçu, em substratos de dunas e de áreas alagáveis, L.H. Fernandes leg. 529, 1 ex., 533, 1 ex., 536, 7 exs, 537, 6 exs, 539, 3 exs, 540, 3 exs, 27/V/95; 543, 11 exs, 547, 1 ex., 548, 1 ex., 550, 3 exs, 551, 10 exs, 552, 3 exs, 553, 5 exs, 555, 2 exs, 23/VI/ 95; 557, 1 ex., 559, 2 exs, 567, 2 exs, 568, 2 exs, 20/VII/95; 587, 2 exs, 588, 1 ex., 589, 1 ex., 590, 1 ex., 31/VIII/95; 592, 2 exs, 
593, 4 exs, 594, 1 ex., 596, 2 exs, 597, 3 exs, 598, 4 exs, 600, 1 ex., 601, 19 exs, 603, 7 exs, 27/IX/95; 610, 6 exs, 611, 5 exs, 612, 2 exs, 613, 5 exs, 614, 4 exs, 616, 3 exs, 619, 2 exs, 27/X/ 95; 627, 1 ex., 629, 1 ex., 630, 3 exs, 631, 6 exs, 633, 1 ex., 24/ XII/95; 637, 5 exs, 638, 7 exs, 642, 1 ex., 643, 5 exs, 644, 1 ex., 647, 1 ex., 16/XII/95; 652, 3 exs, 656, 6 exs, 659, 1 ex., 660, 4 exs, 22/I/96; 679, 1 ex., 29/II/96; 682, 2 exs, 685, 1 ex., 686, 9 exs, 688, 4 exs, 689, 6 exs, 25/III/96; 701, 2 exs, 702, 1 ex., 703, 9 exs, 704, 7 exs, 705, 19 exs, 706, 112 exs, 19/IV/96.

Comentários. Os espécimens de Mesaphorura yosii procedentes da Restinga de Itaipuaçu corresponderam em sua grande maioria às descrições de Rusek (1971) e de Christiansen \& Bellinger (1992). No entanto, os pseudocelos dorsais do segmento abdominal I, nas descrições destes autores, encontramse entre as cerdas $\mathrm{p}_{3}$ e $\mathrm{p}_{4}$, e nos exemplares por nós estudados estão localizados entre as cerdas $\mathrm{p}_{2} \mathrm{e}_{3}$. Isto já havia sido observado também por NAjT et al. (1990) em material procedente da Guiana Francesa e por Oliveira \& Thibaud (1992) em material da Amazônia.

\section{Mesaphorura maricaensis sp. nov.} Figs 1-7

Holótipo fêmea. Brasil, Rio de Janeiro: Maricá, Restinga de Itaipuaçu, em substratos de dunas, L.H. Fernandes leg. em lâmina 707, 19/IV/96. Parátipos da mesma procedência: 535, 2 exs, 27/V/95; 600, 1 ex., 27/IX/95; 626, 1 ex., 24/XI/95; 689, 1 ex., 25/III/96; 703, 65 exs, 707, 55 exs, 19/IV/96.

Comprimento variando de $0,50 \mathrm{~mm}$ a $0,84 \mathrm{~mm}$. Habitus alongado e cilíndrico típico do gênero. Coloração inteiramente branca. Tegumento geral do corpo finamente granulado. Revestimento constituído de cerdas finas e lisas diferenciadas em micro e macroquetas, esparsamente distribuídas conforme figura 1. Pseudocelos circulares com trabéculas concêntricas sugerindo forma estrelar estão dispostos dorsalmente ao longo do corpo segundo a fórmula 11/111/01121.

Região dorsal cefálica com predominância de microquetas. Antenas mais curtas que a diagonal cefálica; antenômero IV composto por pequena vesícula apical simples, um organito subapical, uma microsensila dorso-externa e cinco sensilas subcilíndricos e subiguais; órgão sensorial do antenômero III formado por duas micropapilas alojadas em uma dobra do tegumento, protegidas lateralmente por dois grossos túbulos ligeiramente curvos, direcionados um para o outro, e mais abaixo três cerdas proximais; na face ventral encontra-se um túbulo engrossado; antenômeros I e II com seis e 11 cerdas, respectivamente (Fig. 2). Sem olhos. Órgão pós-antenal formado por cerca de 50-60 vesículas simples dispostas em duas fileiras paralelas e sinuosas ao longo de um eixo transversal (Fig. 3). Peças bucais mastigadoras. Cerdas labrais apresentando fórmula $2 / 5,4,2$, sendo as duas apicais curvas e mais engrossadas que as demais (Fig. 4).

Tergito torácico I de tamanho reduzido e provido de 1+1 pseudocelos, apresentando 8 cerdas medianas; tergitos torácicos II e III portando 1 microsensila lateral e pseudocelos entre as cerdas $\mathrm{m}_{4}$ e $\mathrm{m}_{5}$. Tibiotarsos I, II e III respectivamente com 15 , 15 e 14 cerdas simples e desprovidos de tenent hair. Unha curta e alargada desprovida de dente na crista interna; apêndice empodial ausente.

Tergito abdominal I desprovido de pseudocelos; tergitos abdominais II e III com pseudocelos sob a cerda $\mathrm{p}_{3}$; tergito abdominal IV com pseudocelos entre as cerdas $\mathrm{p}_{3}$ e $\mathrm{p}_{4}$ e entre $\mathrm{p}_{4} \mathrm{e}$ $\mathrm{p}_{5}$; tergito abdominal $\mathrm{V}$ com $2+2$ cerdas entre as macroquetas $\mathrm{a}_{4}$ e pseudocelo localizado sob a cerda s afilada; tergito abdominal VI sem depressões na região anterior e na região posterior presença de dois espinhos anais sobre conspícuas papilas; a relação de comprimento unha:espinho anal apresentou-se 1:0,70. Tubo ventral com $6+6$ cerdas, sendo $2+2$ basais, $2+2$ medianas e $2+2$ apicais. Tenáculo e furca ausentes. Valvas anais representadas na figura 5. Placa genital do macho com cerca de 18 cerdas circungenitais (Fig. 6) e da fêmea com sete cerdas, sendo duas microquetas próximas a fenda genital e cinco cerdas superiores dispostas em semicírculo (Fig. 7).

Etimologia. em referência ao local onde foi coletada, em Maricá.

Comentários. Mesaphorura maricaensis sp. nov. pertence ao grupo sylvatica que inclui as espécies com $2+2$ cerdas entre as macroquetas $\mathrm{a}_{4}$ do segmento abdominal V (Ruser, 1982). Constatamos que dentre as espécies deste grupo, $M$. atlantica Rusek, 1979 é a que mais se aproxima de Mesaphorura maricaensis sp. nov. pelos seguintes caracteres: número de sensilas do antenômero IV, forma do pseudocelo, tibiotarso e unha, quetotaxia cefálica e dos segmentos torácico I e abdominais II a V. Elas diferem pela fórmula pseudocelar, número de vesículas do órgão pós-antenal, quetotaxia dos segmentos torácicos II e III, abdominais I e VI.

Mesaphorura maricaensis sp. nov. se assemelha com $M$. sylvatica Rusek, 1971 no tocante ao número de sensilas do antenômero IV, forma do pseudocelo, quetotaxia do segmento torácico I e abdominais I, III e V. Entretanto, elas diferem quanto à quetotaxia da cabeça, dos segmentos torácicos II e III, dos segmentos abdominais II, IV e VI, fórmula dos pseudocelos, e presença de apêndice empodial.

\section{Brachystomellidae Brachystomella agrosa Wray, 1953}

Material examinado. BRASIL, Rio de Janeiro: Maricá, Restinga de Itaipuaçu, em substratos de dunas e de áreas alagáveis, L.H. Fernandes leg. 527, 21 exs, 528, 6 exs, 529, 15 exs, 530, 17 exs, 531, 1 ex., 532, 8 exs, 533, 2 exs, 535, 4 exs, 537, 18 exs, 538, 1 ex., 540, 4 exs, 27/V/95; 541, 1 ex., 542, 5 exs, 543, 6 exs, 544, 1 ex., 551, 2 exs, 552, 4 exs, 553, 4 exs, 554, 1 ex., 555, 6 exs, 23/VI/95; 557, 15 exs, 558, 3 exs, 559, 1 ex., 560, 4 exs, 567, 3 exs, 570, 4 exs, 20/VII/95; 586, 1 ex., 589, 3 exs, 31/VIII/95; 592, 43 exs, 593, 3 exs, 594, 25 exs, 601, 3 exs, 603, 11 exs, 27/IX/95; 608, 71 exs, 609, 28 exs, 610, 30 exs, 613, 10 exs, 616, 22 exs, 617, 32 exs, 618, 4 exs, 619, 11 exs, 620, 6 exs, 27/X/95; 622, 35 exs, 624, 2 exs, 627, 1 ex., 629, 4 


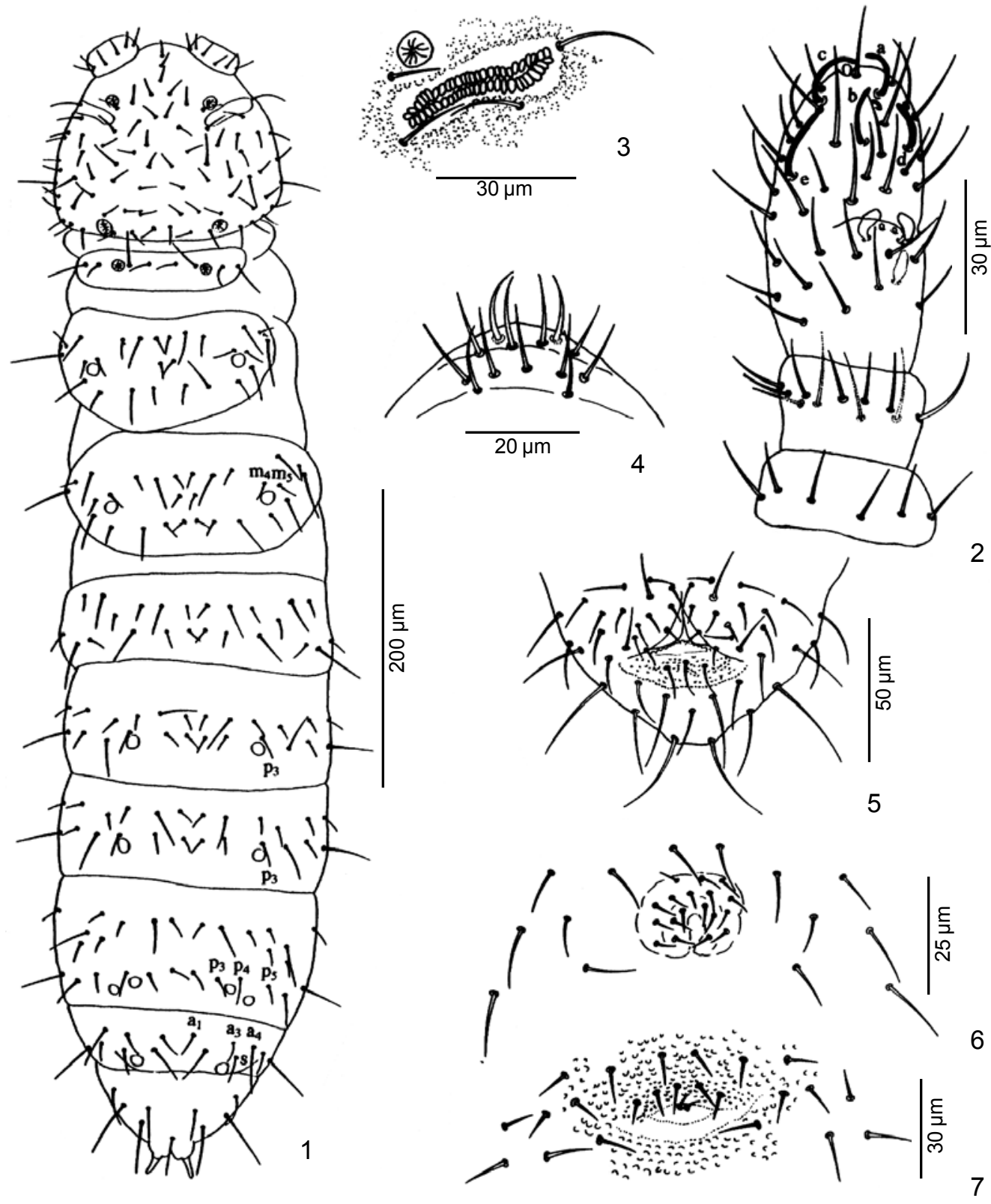

Figuras 1-7. Mesaphorura maricaensis sp. nov.: (1) quetotaxia dorsal; (2) face dorso-lateral da antena; (3) pseudocelo e órgão pósantenal; (4) cerdas labrais; (5) valvas anais; (6) placa genital do macho; (7) placa genital da fêmea.

exs, 632, 5 exs, 634, 4 exs, 635, 8 exs, 24/XI/95; 637, 5 exs, 638, 7 exs, 639, 1 ex., 643, 2 exs, 644, 1 ex., 647, 6 exs, 648, 14 exs, 650, 1 ex., 16/XII/95; 652, 1 ex., 656, 1 ex., 665, 3 exs, 22/I/96; 668, 1 ex., 29/II/96; 682, 13 exs, 683, 2 exs, 684, 6 exs, 686, 2 exs, 691, 1 ex., 25/III/96; 700, 2 exs, 701, 29 exs, 702, 1 ex., 703, 2 exs, 709, 6 exs, 711, 15 exs, 19/IV/96.

Comentários. Brachystomella agrosa, comum em toda a região neotropical e abundante no Brasil, tem sido encontrada nos ambientes mais diversos. O estudo dos exemplares de $B$. agrosa procedentes da restinga de Itaipuaçu mostrou-se concordante com as descrições e ilustrações de CASSAGNAU \& RAPOPORT (1962), Masoud \& Thibaud (1980), Najt et al. (1990) e Mendonça \& ARLÉ (1992). Apesar da grande homogeneidade constatada na quetotaxia e ilustrada por Massoud \& Thibaud (1980) nos

Revista Brasileira de Zoologia 21 (1): 15-25, março 2004 
espécimens procedentes das Pequenas Antilhas, averiguou-se apenas que as cerdas centrais cefálicas do material estudado mostraram-se mais numerosas.

\section{Brachystomella contorta Denis, 1931}

Material examinado. BRASIL, Rio de Janeiro: Maricá, Restinga de Itaipuaçu, em substratos de dunas, L.H. Fernandes leg. 526, 9 exs, 27/V/95; 545, 1 ex., 23/VI/95; 707, 2 exs, 19/IV/96.

Comentários. Os exemplares procedentes da restinga de Itaipuaçu enquadram-se perfeitamente com a descrição e ilustrações de Denis (1931) e Christiansen \& Bellinger (1992).

\section{Brachystomella ceciliae sp. nov. Figs 8-13}

Holótipo fêmea. BrasiL, Rio de Janeiro: Maricá, Restinga de Itaipuaçu, em substratos de dunas e de áreas alagáveis, L.H. Fernandes leg. em lâmina 664, 22/I/96. Parátipos da mesma procedência em lâminas e em álcool: 534, 1 ex., 27/V/95; 541, 5 exs, 544, 1 ex., 550, 1 ex., 23/VII/95; 558, 4 exs, 566, 2 exs, 568, 4 exs, 20/VII/95; 588, 3 exs, 31/VIII/95; 591, 2 exs, 27/IX/ 95; 608, 8 exs, 612, 5 exs, 616, 3 exs, 617, 6 exs, 619, 1 ex., 620, 3 exs, 27/X/95; 621, 5 exs, 634, 9 exs, 635, 2 exs, 24/XI/95; 637, 2 exs, 16/XII/95; 653, 1 ex., 654, 3 exs, 664, 1 ex., 22/I/96; 668, 2 exs, 29/II/96; 682, 2 exs, 25/III/96.

Comprimento variando de $0,40 \mathrm{~mm}$ a $0,56 \mathrm{~mm}$. Habitus ovóide e robusto. Coloração cinza claro com antenas, patas e face ventral mais claras. Tegumento mediamente granuloso. Revestimento de cerdas finas e lisas, cerdas sensoriais medindo cerca de duas vezes o comprimento das demais e obedecendo a seguinte fórmula por meio-tergito: 022/11111; quetotaxia dorsal conforme figura 8 .

Antenas mais curtas que a diagonal cefálica; antenômero IV constituído por vesícula apical unilobada ou bilobada, um organito subapical alojado em fosseta, uma microsensila dorso-externa, cinco sensilas subcilíndricas; órgão sensorial do antenômero III formado por duas microsensilas dobradas, voltadas na mesma direção e protegidas por dois tubos olfativos subcilíndricos de tamanhos diferentes, uma pequena sensila na face ventral; antenômeros I e II com sete e 12 cerdas, respectivamente (Fig. 9). Com $8+8$ olhos em placa ocular fortemente pigmentada. Órgão pós-antenal constituído de quatro lóbulos em roseta, medindo aproximadamente o diâmetro de uma cornéula (Fig. 10). Maxila globulosa com oito dentes (Fig. 11).

Tergito torácico I com $3+3$ cerdas; tergito torácico II com $4+4$ cerdas centrais entre as sensoriais; tergito torácico III com $3+3$ cerdas centrais entre as sensoriais. Tibiotarsos I, II e III respectivamente com 18, 18 e 17 cerdas; 1 tenent hair longo e capitado. Unha longa e fina com um dente no terço médio da crista interna; apêndice empodial ausente (Fig. 12).

Cerdas dos tergitos abdominais IV ao VI mais longas que as demais; tergito abdominal $\mathrm{V}$ com $3+3$ cerdas centrais entre as sensoriais. Tubo ventral com $3+3$ cerdas. Tenáculo com $3+3$ dentes. Furca normalmente desenvolvida; manúbrio com cerca de 22 cerdas, sendo $1+1$ entre as basais, $1+1$ entre as centrais e $1+1$ entre as apicais maiores que as demais; dens com seis cerdas na face dorsal; mucro alongado com ápice pontudo medindo aproximadamente metade do comprimento da dens, lamelas externa e interna estreitas (Fig. 13).

Etimologia. Nome em homenagem à Maria Cecília de Chiara Moço pelo auxílio durante as coletas.

Comentários. Brachystomella ceciliae sp. nov. pertence ao grupo stachi juntamente com as espécies $B$. unguilongus Najt \& Thibaud, 1988 e B. coatesi Weiner \& Najt, 1991, por apresentarem em comum o número de olhos, órgão pós-antenal, unhas longas e finas, número de cerdas da dens e fórmula sensorial dos tergitos.

Brachystomella ceciliae sp. nov. assemelha-se a B. stachi Mills, 1934 quanto à quetotaxia dos segmentos abdominais III e IV-VI, divergindo no entanto, quanto ao número de sensilas do antenômero IV, número e disposição dos dentes na maxila, quetotaxia cefálica, torácica e do segmento abdominal III.

Brachystomella ceciliae sp. nov. também apresentou semelhanças com a espécie $B$. unguilongus no tocante ao número de cerdas do antenômero I, número de sensilas do antenômero IV, número de dentes da maxila, número de cerdas do tibiotarsos I-III, quetotaxia torácica. Difere da mesma pela quetotaxia cefálica e abdominal, número de cerdas do antenômero II, vesícula apical do antenômero IV e forma do mucro.

Existem semelhanças entre Brachystomella ceciliae sp. nov. e $B$. coatesi no que diz respeito ao número de cerdas dos antenômeros I e II, órgão sensorial do antenômero III, vesícula apical do antenômero IV, número de cerdas dos tibiotarsos I a III, presença de dente na crista interna da unha e forma do mucro. Elas se distinguem porém, pela quetotaxia cefálica, do segmento torácico I e abdominal IV, disposição e número de dentes na maxila.

\section{Brachystomellides compositus Arlé, 1959}

Material examinado. BRASIL, Rio de Janeiro: Maricá, Restinga de Itaipuaçu, em substratos de dunas, L.H. Fernandes leg. 550, 1 ex., 23/VII/95.

Comentários. O único exemplar coletado corresponde perfeitamente à descrição original.

\section{Rapoportella pitomboi Mendonça \& Fernandes, 1995}

Material examinado. BRASIL, Rio de Janeiro: Maricá, Restinga de Itaipuaçu, em substratos de dunas e de áreas alagáveis, L.H. Fernandes leg. 531, 1 ex., 534, 1 ex., 535, 7 exs, 27/V/95; 613, 2 exs, 27/X/95; 631, 16 exs, 632, 11 exs, 634, 1 ex., 24/XI/ 95; 643, 2 exs, 647, 8 exs, 16/XII/95; 656, 1 ex., 658, 2 exs, 22/ I/96; 707, 5 exs, 19/IV/96.

Comentários. Os exemplares de Rapoportella pitomboi coletados na Restinga de Itaipuaçu apresentaram uma coloração azulada, diferente da coloração rosada exibida pelo exemplares oriundos de Itatiaia (Minas Gerais) a $2000 \mathrm{~m}$ de altitude. 

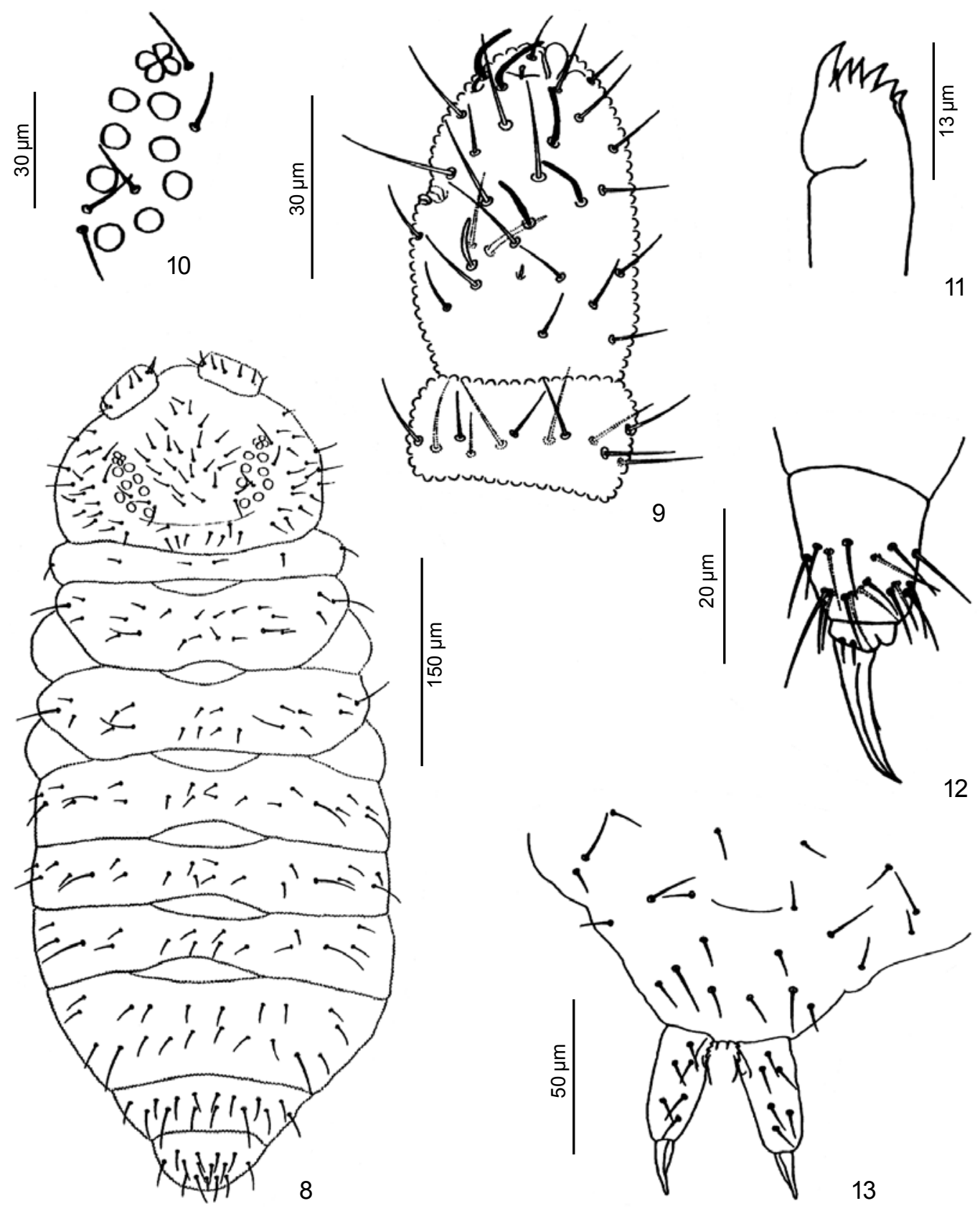

Figuras 8-13. Brachystomella ceciliae sp. nov.: (8) quetotaxia dorsal; (9) face dorsal dos antenômeros II ao IV; (10) olhos e órgão pósantenal; (11) maxila; (12) tibiotarso e unha da pata I; (13) face dorsal da furca.

\section{Maricaella duna Mendonça \& Fernandes, 1997}

Material examinado. Brasil, Rio de Janeiro: Maricá, Restinga de Itaipuaçu, em substratos de dunas, L.H. Fernandes leg. 535, 2 exs, 27/V/95; 547, 1 ex., 23/VI/95; 596, 1 ex., 597, 3

Revista Brasileira de Zoologia 21 (1): 15-25, março 2004 exs, 598, 1 ex., 599, 1 ex., 27/IX/95; 614, 1 ex., 27/X/95; 627, 1 ex., 24/XI/95; 705, 1 ex., 19/IV/96.

Comentários. Maricaella duna, espécie de ocorrência restrita para a região, mostrou-se concordante com a descrição original. 


\section{Neanuridae Friesea reducta Denis, 1931}

Material examinado. BRAsil, Rio de Janeiro: Maricá, Restinga de Itaipuaçu, em substratos de dunas, L.H. Fernandes leg. 527, 38 exs, 528, 18 exs, 529, 5 exs, 530, 2 exs, 531, 26 exs, 532, 2 exs, 533, 7 exs, 534, 12 exs, 27/V/95; 541, 28 exs, 542, 22 exs, 546, 76 exs, 547, 16 exs, 548, 21 exs, 549, 15 exs, 550, 29 exs, 23/VI/95; 557, 5 exs, 558, 1 ex., 560, 2 exs, 561, 17 exs, 562, 8 exs, 563, 13 exs, 564, 17 exs, 20/VII/95; 585, 3 exs, 31/ VIII/95; 592, 15 exs, 593, 3 exs, 594, 31 exs, 596, 5 exs, 597, 2 exs, 598, 13 exs, 599, 9 exs, 600, 9 exs, 27/IX/95; 608, 4 exs, 610, 30 exs, 611, 2 exs, 612, 4 exs, 613, 1 ex., 614, 3 exs, 615, 7 exs, 27/X/95; 625, 36 exs, 626, 1 ex., 627, 15 exs, 628, 6 exs, 629, 6 exs, 630, 2 exs, 24/XI/95; 637, 1 ex., 638, 5 exs, 641, 1 ex., 642, 3 exs, 643, 9 exs, 644, 2 exs, 16/XII/95; 652, 1 ex., 654, 4 exs, 659, 1 ex., 660, 8 exs, 22/I/96; 670, 2 exs, 29/II/96; 685, 1 ex., 687, 2 exs, 689, 2 exs, 690, 2 exs, 25/III/96; 700, 13 exs, 702, 7 exs, 703, 20 exs, 704, 12 exs, 705, 6 exs, 706, 13 exs, 707, 64 exs, 19/IV/96.

Comentários. Na descrição de Denis (1931), baseada em exemplares coletados na Costa Rica, os tenent hairs dos tibiotarsos I a III estão ausentes, ao passo que nos exemplares da Restinga de Itaipuaçu foram observados em número de 4, 5 e 5 em cada tibiotarso, fato este citado anteriormente por Massoud \& Thibaud (1980) em material procedente das Pequenas Antilhas.

\section{Pseudachorutes difficilis Denis, 1931}

Material examinado. Brasil, Rio de Janeiro: Maricá, Restinga de Itaipuaçu, em substratos de dunas e de áreas alagáveis, L.H. Fernandes leg. 527, 7 exs, 530, 3 exs, 537, 14 exs, 540, 5 exs, 27/V/95; 541, 1 ex., 542, 1 ex., 546, 1 ex., 551, 12 exs, 552, 1 ex., 553, 6 exs, 555, 3 exs, 23/VI/95; 566, 2 exs, 568, 5 exs, 20/VII/95; 586, 1 ex., 587, 1 ex., 588, 5 exs, 589, 15 exs, 590, 19 exs, 31/VIII/95; 597, 9 exs, 601, 1 ex., 603, 3 exs, 27/IX/95; 610, 1 ex., 616, 3 exs, 617, 2 exs, 618, 5 exs, 619, 1 ex., 27/X/95; 634, 1 ex., 635, 17 exs, 24/XI/95; 637, 4 exs, 649, 2 exs, 16/XII/95; 664, 1 ex., 665, 2 exs, 22/I/96; 704, 1 ex., 19/ IV/96.

Comentários. Os exemplares de Pseudachorutes difficilis coletados na Restinga de Itaipuaçu concordam perfeitamente com a revisão do material-tipo feita por NAJT et al. (1991).

\section{Aethiopella littoralis Fernandes \& Mendonça, 2002}

Material examinado. Brasil, Rio de Janeiro: Maricá, Restinga de Itaipuaçu, em substratos de dunas e de áreas alagáveis, L.H. Fernandes leg. 627, 1 ex, 24/XI/1995; 527, 1 ex., 27/V/1995; 609, 1 ex., 27/X/1995; 610, 3 exs, 27/X/1995; 619, 1 ex., 27/X/1995; 627, 1 ex., 24/XI/1995; 637, 1 ex., 16/XII/ 1995; 652, 1 ex., 22/I/1996; 655, 1 ex., 22/I/1996; 684, 3 exs, 25/III/1996; 701, 1 ex., 19/IV1996.
Comentários. Aethiopella littoralis é uma espécie de ocorrência até o momento restrita para a região.

\section{Arlesia intermedia sp. nov. Figs 14-19}

Holótipo macho. Brasil, Rio de Janeiro: Maricá, Restinga de Itaipuaçu, em substratos de dunas e de áreas alagáveis, L.H. Fernandes leg., em lâmina 632, 24/XI/95. Parátipos da mesma procedência: 533, 1 ex., 27/V/95; 629, 3 exs, 24/XI/95; 694, 3 exs, 25/III/96; 707, 1 ex., 711, 6 exs, 19/IV/96.

Comprimento variando de $0,98 \mathrm{~mm}$ a $1,6 \mathrm{~mm}$. Habitus típico de Pseudachorutinae com paratergitos arredondados. Coloração cinza claro com face ventral, patas, cone bucal e extremidade das antenas mais claros. Tegumento fortemente granuloso. Revestimento formado por cerdas finas e lisas; cerdas sensoriais longas e lisas; fórmula sensorial por meio-tergito: 022/11111; quetotaxia dorsal conforme figura 14.

Antenas mais curtas que a diagonal cefálica; antenômero IV composto por vesícula apical trilobada, quatro sensilas subcilíndricas entre cerdas de comprimentos variados; órgão sensorial do antenômero III constituído de dois túbulos retos e protegidos por duas sensilas de guarda, uma pequena sensila na face ventral (Fig. 15); antenômeros I e II com 10 e 11 cerdas, respectivamente. Com $5+5$ olhos em placa ocular levemente pigmentada. Órgão pós-antenal ausente, mas apresentando granulação mais fina no local correspondente. Mandíbula com cinco dentes, sendo dois apicais subiguais, um mediano grosso e provido de pequeno dente e um basal longo e fino (Fig. 16). Maxila estiliforme com duas lamelas soldadas (Fig. 17). Cerdas labiais conforme figura 18. Cerdas labrais apresentando fórmula $2 / 3,5,2$.

Tergito torácico I com $3+3$ cerdas; tergito torácico II com $3+3$ cerdas centrais entre as sensoriais proximais e portando 1+1 microsensila na lateral; tergito torácico III com $3+3$ cerdas centrais entre as sensoriais proximais. Tibiotarsos I, II e III respectivamente com respectivamente 19, 19 e 18 cerdas e desprovidos de tenent hairs. Unha com um dente na crista interna. Apêndice empodial ausente.

Tergitos abdominais I-IV com $3+3$ cerdas entre as sensoriais; tergito abdominal $\mathrm{V}$ com $2+2$ cerdas entre as sensoriais; tergito abdominal VI pouco visível dorsalmente. Tubo ventral com $3+3$ cerdas. Tenáculo com $3+3$ dentes. Furca normalmente desenvolvida. Dens com seis cerdas na face dorsal e mucro reto com extremidade ligeiramente curva (Fig. 19); relação do comprimento dens: mucro $=1: 0,50$. Placa genital do macho com cerca de 26 cerdas circungenitais.

Etimologia. Nome alusivo à proximidade morfológica com Arlesia albipes e A. arleana.

Comentários. Arlesia intermedia sp. nov. concorda com A. albipes e A. arleana em relação à maior parte dos caracteres. No entanto afasta-se de $A$. albipes quanto à quetotaxia e o número de dentes da mandíbula; e de $A$. arleana quanto à coloração, forma da mandíbula e no número de sensilas da antena.

Revista Brasileira de Zoologia 21 (1): 15-25, março 2004 

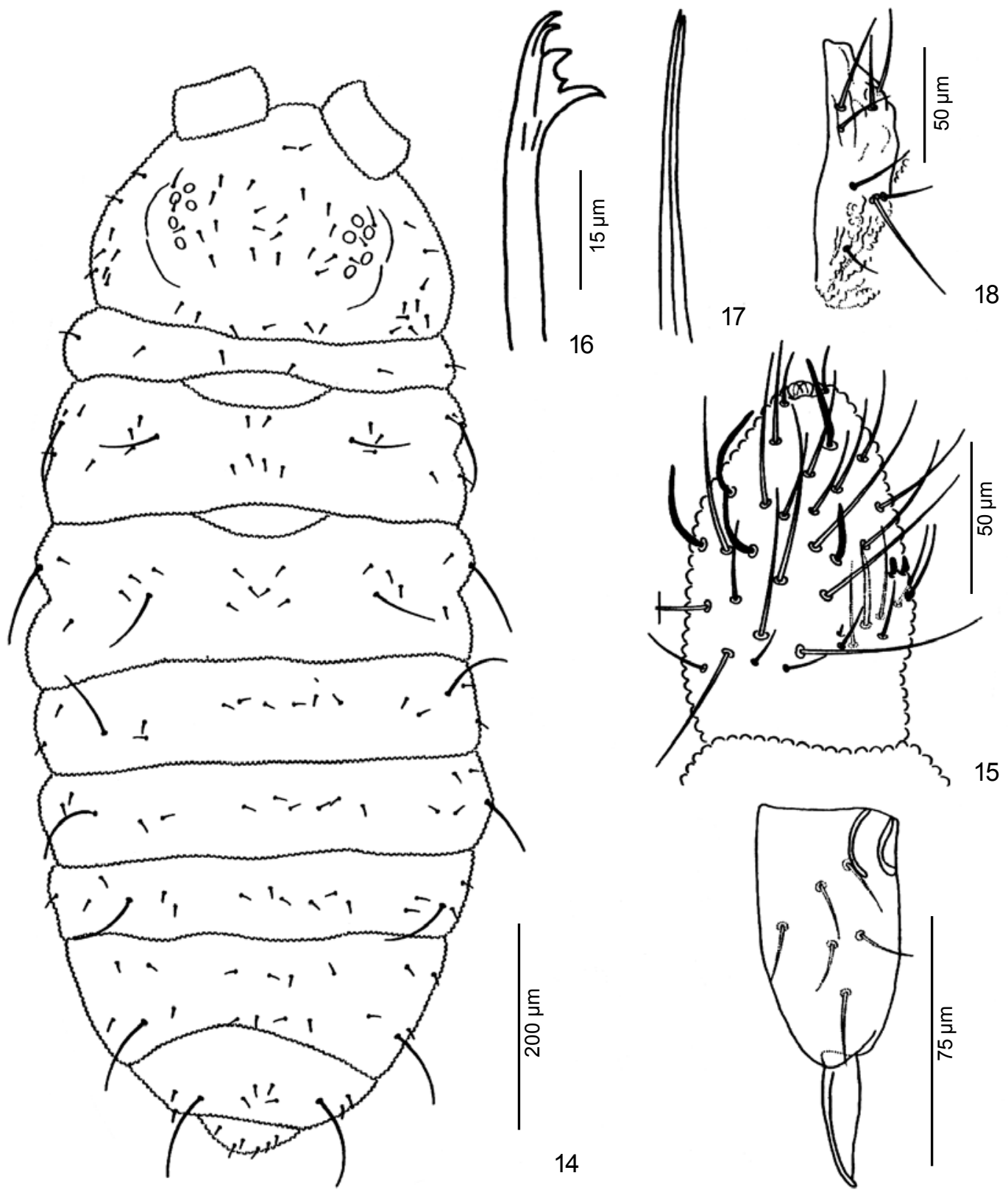

17

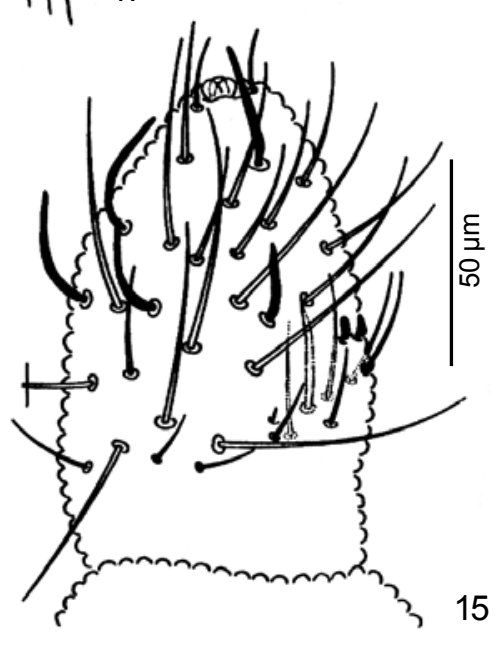

4

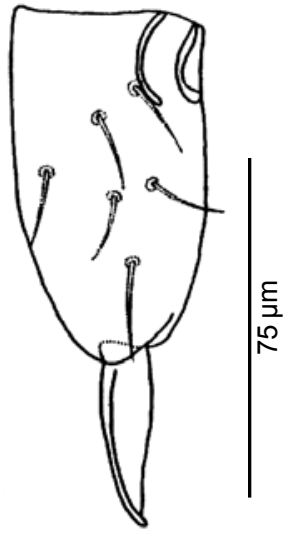

Figuras 14-19. Arlesia intermedia sp. nov.: (14) quetotaxia dorsal; (15) face dorso-lateral do antenômero III-IV; 16- mandíbula; (17) maxila; (18) cerdas labiais; (19) face dorsal da dens e mucro.

\section{Micranurida fluminensis sp. nov.}

Figs $20-26$

Holótipo fêmea. BRASIL, Rio de Janeiro: Maricá, Restinga de Itaipuaçu, em substratos de dunas e de áreas alagáveis, L.H. Fernandes leg., em lâmina 707, 19/IV/96. Parátipos da mesma procedência: 535, 1 ex., 27/V/95; 631, 1 ex., 24/XI/95; 707, 2 exs, 19/IV/96.
Comprimento variando de $0,39 \mathrm{~mm}$ a $0,52 \mathrm{~mm}$. Habitus robusto. Coloração branca, com pigmento azul escuro disperso apenas na área dos olhos. Tegumento mediamente granuloso. Revestimento formado por cerdas finas e lisas; cerdas sensoriais um pouco mais longas que as cerdas proximais e obedecendo à seguinte fórmula por meio-tergito: 022/11111; quetotaxia dorsal conforme figura 20 .

Revista Brasileira de Zoologia 21 (1): 15-25, março 2004 


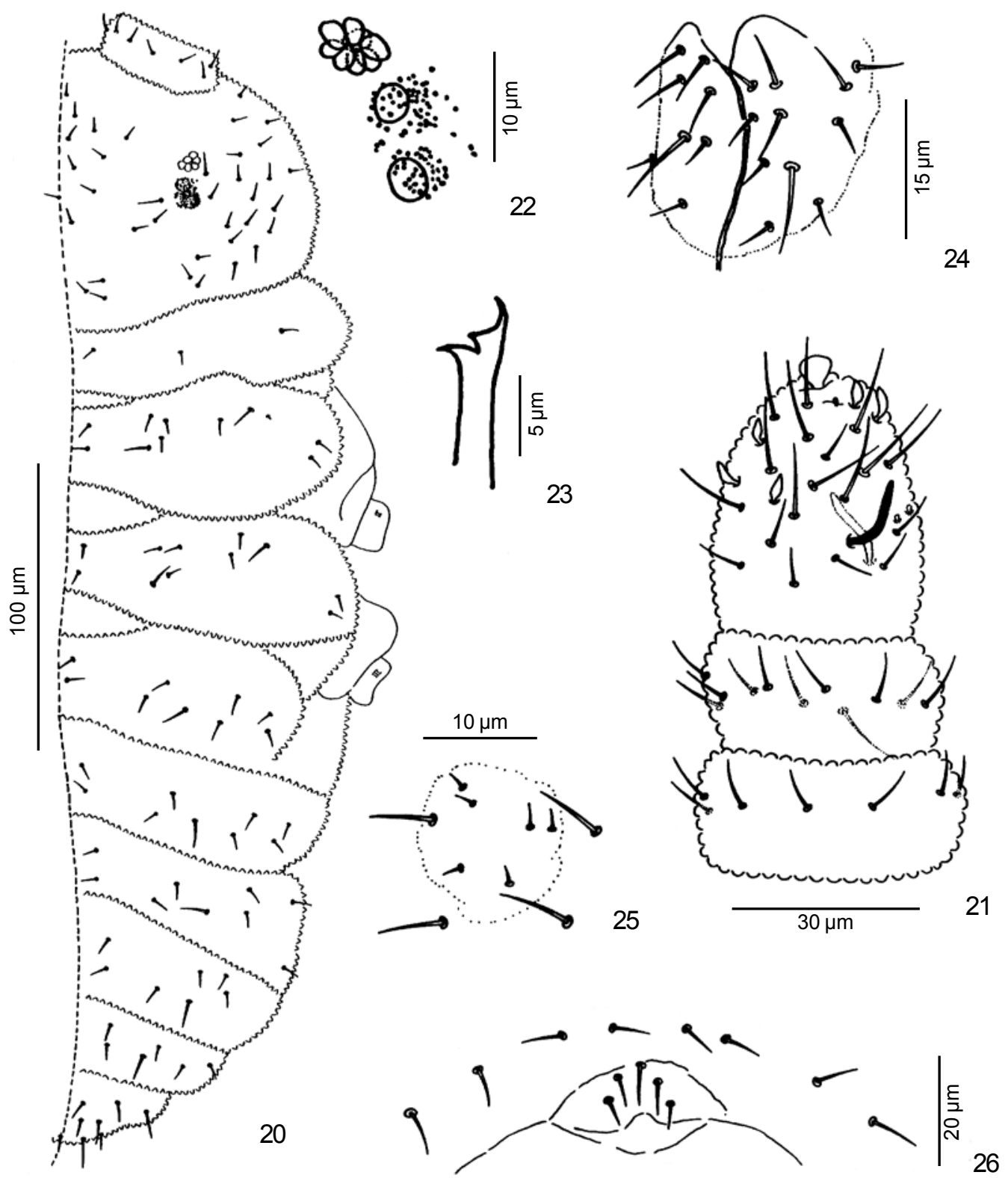

Figuras 20-26. Micranurida fluminensis sp. nov.: (20) quetotaxia dorsal; (21) face dorso-lateral da antena; (22) olhos e órgão pósantenal; (23) mandíbula; (24) cerdas labiais; (25) área remanescente da furca; (26) placa genital da fêmea.

Antenas mais curtas que a diagonal cefálica; antenômero IV composto por vesícula apical unilobada, 1 organito subapical alojado em fosseta, uma microsensila dorso-externa, cinco sensilas em forma de chama de vela, sendo duas dorso-externas e três dorso-internas; órgão sensorial do antenômero III formado por duas microsensilas arredondadas e protegidos por duas sensilas de guarda subcilíndricas, uma pequena sensila na face ventral; antenômeros I e II com 7 e 11 cerdas, respecti- vamente (Fig. 21). Com 2+2 olhos; órgão pós-antenal constituído de seis a sete lóbulos e medindo cerca de duas vezes o diâmetro de uma cornéula (Fig. 22). Mandíbula com três dentes, sendo o apical fino e curto (Fig. 23), maxila estiliforme. Cerdas labiais conforme figura 24 .

Tergito torácico I com $3+3$ cerdas; Tergito torácico II com $2+2$ cerdas centrais entre as sensoriais proximais e portando 1+1 microsensila na lateral; tergito torácico III com $2+2$ cerdas 
centrais entre as sensoriais proximais. Tibiotarsos I, II e III respectivamente com 13, 13 e 12 cerdas e desprovidos de tenent hairs. Unha sem dente na crista interna. Apêndice empodial ausente.

Tergitos abdominais I-V com $2+2$ cerdas centrais entre as sensoriais. Tubo ventral com $4+4$ cerdas. Furca e tenáculo ausentes; zona furcal portando $3+3$ microquetas protegidas por $2+2$ cerdas (Fig. 25). Placa genital da fêmea com cinco cerdas, sendo duas próximas à fenda genital e três superiores dispostas em semicírculo (Fig. 26).

Etimologia. Nome em alusão à sua origem no Estado do Rio de Janeiro.

Comentários. O gênero Micranurida Börner, 1901, de distribuição cosmopolita, é registrado pela primeira vez para o Brasil.

Micranurida candida Cassagnau, 1952 é a espécie que mais se aproxima de Micranurida fluminensis sp. nov. pelos seguintes caracteres: vesícula apical do antenômero IV, número de olhos, número de lóbulos do pós-antenal. No entanto, afastase da mesma apenas em relação ao número de sensilas do antenômero IV.

Embora Micranurida fluminensis sp. nov. apresente semelhanças também com M. wladimiri Najt \& Rubio, 1978 no tocante ao número de olhos, número de vesículas do órgão pósantenal, vesícula apical e número de sensilas do antenômero IV e órgão sensorial do antenômero III; difere da mesma quanto ao número de cerdas nos tibiotarsos I-III e quanto à quetotaxia da base da cabeça.

A nova espécie aproxima-se também de $M$. lanceolata Najt \& Weiner, 1985 quanto ao número de olhos, número de vesículas do órgão pós-antenal, vesícula apical e número de sensilas do antenômero IV, órgão sensorial do antenômero III, número de cerdas dos antenômeros I e II, fórmula sensorial por meiotergito, quetotaxia cefálica, do segmento torácico I e dos segmentos abdominais I-IV e VI. No entanto, Micranurida fluminensis sp. nov. difere essencialmente de M. lanceolata pelas cerdas sensoriais em forma de lança exibidas por esta última.

\section{Hylaeanura infima (Arlé, 1959)}

Material examinado. Brasil, Rio de Janeiro: Maricá, Restinga de Itaipuaçu, em substratos de dunas e de áreas alagáveis, L.H. Fernandes leg. 537, 6 exs, 27/V/95; 551, 2 exs, 553, 5 exs, 23/VI/95; 568, 1 ex., 20/VII/95; 597, 3 exs, 601, 2 exs, 603, 4 exs, 27/IX/95; 618, 4 exs, 619, 1 ex., 27/X/95; 632, 1 ex., 24/XI/95; 647, 1 ex., 16/XII/95; 704, 20 exs, 19/IV/96.

Comentários. Os exemplares de Hylaeanura infima procedentes da Restinga de Itaipuaçu concordaram em sua totalidade com as descrições e ilustrações de ArLé (1959), ArLé (1966b) e Thibaud \& Massoud (1983).

\section{DISCUSSÃO}

Das 20 espécies de Poduromorpha encontradas na Restinga de Itaipuaçu, foram registradas pela primeira vez para o Brasil Xenylla maritima, Acherontiella globulata, Onychiurus cf. mariapetrae, Friesea reducta, Pseudachorutes difficilis, e as espécies dos gêneros Doutnacia Rusek, 1974 e Micranurida Börner, 1901. Das espécies já conhecidas no Brasil Mesaphorura amazonica, Rapoportella pitomboi e Hylaeanura infima, tiveram sua área de ocorrência ampliada para o Rio de Janeiro. Brachystomella contorta e Brachystomellides compositus, espécies já encontradas no Rio de Janeiro, foram registradas pela primeira vez em Maricá. Mesaphorura yosii, Maricaella duna, Brachystomella agrosa e Aethiopella littoralis já haviam sido registradas anteriormente para a área de estudo. A descrição de quatro novas espécies: Mesaphorura maricaensis sp. nov.; Brachystomella ceciliae sp. nov.; Arlesia intermedia sp. nov.; Micranurida fluminensis sp. nov., bem como o registro de ocorrência de outras 12 espécies na região mostram a falta de exploração científica e refletem a elevada biodiversidade deste ecossistema costeiro.

\section{AGRADECIMENTOS}

Agradecemos ao Conselho Nacional de Desenvolvimento Científico e Tecnológico (CNPq), pela concessão de bolsas de estudos; à Fundação Carlos Chagas Filho de Amparo a Pesquisa do Estado do Rio de Janeiro (FAPERJ) e à Fundação Universitária José Bonifácio (FUJB) pelo auxílio financeiro; e ao Prof. Dr. Guilherme Muricy pela leitura crítica do manuscrito.

\section{REFERÊNCIAS BIBLIOGRÁFICAS}

Arbea, J.I. \& R. Jordana. 1991. Colémbolos de Navarra (Norte de la Península Ibérica). I. Orden Poduromorpha (Collembola). Publicaciones de Biologia de la Universidad de Navarra, Série Zoologica, Pamplona, 22: 1-149.

ArLé, R. 1959. Collembola Arthropleona do Brasil Oriental e Central. Arquivos do Museu Nacional do Rio de Janeiro, Rio de Janeiro, 49: 155-211.

- 1966a. Un nouveau collembole marin du littoral brésilien. Revista Brasileira de Biologia, Rio de Janeiro, 26 (4): 367-372.

. 1966b. Collemboles d'Amazonie. I. Poduromorphes nouveaux ou peu connus et notes biologiques sur Neotropiella carli (Denis). Boletim do Museu Paraense Emílio Goeldi, Nova Série Zoologia, Belém, 60: 1-19.

Bellinger, P.F.; K.A. Christiansen \& F. Janssens. 1996-2003. Checklist of the Collembola of the World. http://www.collembola. org

Borner, C. 1913. Die familien der Collembolen. Zoologischer Anzeiger, Leipzig, 41: 315-322.

Cassagnau, P. \& E.H. Rapoport. 1962. Collemboles d'Amerique du Sud. I. Poduromorphes. Biologie d'Amerique Australe, Paris, 1: 139-184.

Christiansen, K. \& P.F. Bellinger. 1980. The Collembola of Nort America north of the Rio Grande, a analysis taxonomic. Iowa: Grinnell College. $1322 \mathrm{p}$.

. 1992. Insects of Hawaii. Collembola. Hawaii, Hawaii University Press, vol.15, VIII+445p. 
DenIS, J.R. 1931. Contributo alla conoscenza del Microgenton di Costa Rica. II. Collemboles de Costa Rica avec une contribuition au species de l'ordre. Bolletino del Laboratoria di Zoologia generale ed agraria della Facoltà agraria di Portici 25: 69-170.

Dorvillé, L.F.M. 1995. Composição e aspectos da biologia da fauna de mosquitos (Diptera, Culicidae) da restinga de Barra de Maricá (RJ). Revista Brasileira de Entomologia, São Paulo, 39 (1): 203-219.

Fernandes, L.H. \& M.C. Mendonça. 2002. Duas novas espécies de Pseudachorutinae (Collembola, Neanuridae) do Brasil. Boletim do Museu Nacional, Nova Série, Zoologia, Rio de Janeiro, 496: 1-8.

Gama, M.M. da. 1964. Colêmbolos de Portugal Continental. Memórias e Estudos do Museu Zoológico da Universidade de Coimbra, Coimbra, 292: 1-252.

LuCiañez, M.J. \& J.C. Simón. 1993. Filogenia de los Tullbergiinae Bagnall, 1935 de la Peninsula Iberica (Collembola, Onychiuridae). Nouvelle Revue d'Entomologie, N.S., Paris, 10 (2): 187-200.

Massoud, Z. 1967. Monographie des Neanuridae, Collemboles Poduromorphes à pièces buccales modifièes. Biologie de l'Amerique Australe, Paris, 3: 1-399.

Massoud, Z. \& J.-M. Thibaud. 1980. Les Collemboles des Petites Antilles II.- Neanuridae. Revue d'Écologie et Biologie du Sol, Paris, 17 (4): 591-605.

Mendonça, C. De \& R. Arlé. 1992. Variação quetotáxica em Brachystomella agrosa Wray, 1953 (Collembola, Neanuridae). Boletim do Museu Nacional, Nova Série, Zoologia, Rio de Janeiro, 351: 1-9.

Mendonça, C. DE \& L.H. Fernandes. 1997. A new genus of Brachystomellinae from Brazil (Collembola: Neanuridae). Boletim do Museu Nacional, Nova Série, Zoologia, Rio de Janeiro, 379: 1-7.

. 1995. Nova espécie de Rapoportella Ellis \& Bellinger, 1973 do Brasil (Collembola: Neanuridae). Boletim do Museu Nacional, Nova Série, Zoologia, Rio de Janeiro, 360: $1-6$.

Najt, J.; J.-M. Thibaud \& S. Jacquemart. 1991. Les Collemboles (Insecta) de l'Archipel des Galápagos I. Poduromorpha. Entomologie, Bélgica, 61: 149-166.

Najt, J.; J.-M. Thibaud \& W. Weiner. 1990. Collemboles (Insecta) Poduromorphes de Guyane française. Bulletin du Museu National d'Histoire Naturelle, $4^{\mathrm{e}}$ sér., section A, Paris, 12 (1): 95-121.

Nimer, E. 1977. Clima, p. 51-89. In: IBGE (Ed.). Geografia do
Brasil. Região Sudeste. Rio de Janeiro, IBGE, vol. 3.

Oliveira, E. \& J.-M. Thibaud. 1992. Notes sur les collemboles de l'Amazonie, Brésil. 1. Hypogastruridae et Onychiuridae, avec la description de deux espèces nouvelles (Collembola). Opuscula zoologica fluminensia, Suíça, 95: 1-8.

Palacios-Vargas, J.G. \& B.E. Mejia. 1988. Colémbolos de Jalisco, México, com descripción de nuevas Friesea (Collembola: Neanuridae) y clave para determinar especies. Folia Entomologica Mexicana, México, 77: 19-31.

Ruser, J. 1971. Zur Taxonomie der Tullbergia (Mesaphorura) krausbaueri (Börner) und ihrern Verwandten (Collembola). Acta Entomologica Bohemoslovaca, República Tcheca, 68: 188-206.

. 1982. European Mesaphorura species of the sylvaticagroup (Collembola, Onychiuridae, Tullbergiinae). Acta Entomologica Bohemoslovaca, República Tcheca, 79: 14-30.

SALMON, J.T. 1964. An Index to the Collembola. Bulletin of the Royal Society of New Zealand, New Zealand, 7 (1-3): 1651.

Silva, J.G. DA \& A.S. DE Oliveira. 1989. A vegetação de Restinga no Município de Maricá, RJ. Acta Botânica Brasileira, São Paulo, 3 (2): 253-272.

Suguio, K. \& M.G. Tessler. 1984. Planícies de cordões litorâneos quaternários do Brasil: origem e nomenclatura, p.15-25. In: L.D. de Lacerda; R. Cerqueira \& B. Turce (Eds). Restingas: Origem, Estrutura, Processos. Niterói, CEUFF, 475p.

Thibaud, J.-M. 1990. Révision du genre Acherontiella Absolon, 1913 (Insecta, Collembola). Bulletin du Museu National d'Histoire Naturelle, $4^{\mathrm{e}}$ sér., section A, Paris, 12 (2): 401-414. . 1993. Les Collemboles des Petites Antilles. VI. Interstitiels terrestres et marins. Revue Française d'Entomologie, N.S., Paris, 15 (2): 69-80.

Thibaud, J.-M. \& Z. Massoud. 1979. Les Collemboles des Petites Antilles I. - Hypogastruridae et Onychiuridae. Revue d'Écologie et Biologie du Sol, Paris, 16 (4): 547-567.

. 1983. Les Collemboles des Petites Antilles III. - Neanuridae (Pseudachorutinae). Revue d'Écologie et Biologie du Sol, Paris, 20 (1): 111-129.

Thibaud, J.-M. \& J.G. Palacios-Vargas. 1999. Brazilian Collembola from littoral sand with description of Austrogastrura gen. $\mathrm{n}$. and Isotomodes carioca sp. n. [Hypogastruridae; Isotomidae]. Revue Française d'Entomologie, N.S., Paris, 21 (1): 25-31.

Weiner, W.M. 1996. Generic revision of Onychiurinae (Collembola: Onychiuridae) with a cladistic analysis. Annales de la Societé Entomologique de France, N.S., Paris, 32 (2): 163-200.

Recebido em 10.X.2003; aceito em 14.I.2004. 